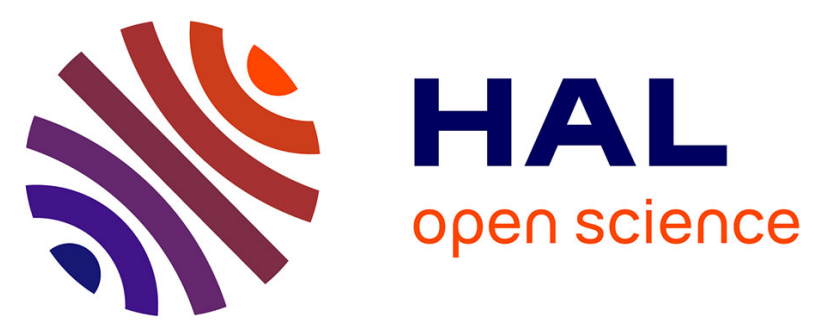

\title{
Rex Fundator. Les interventions royales dans les collèges universitaires de Paris, Oxford et Cambridge (XIVe-XVe siècle)
}

\author{
Thierry Kouamé
}

\section{- To cite this version:}

Thierry Kouamé. Rex Fundator. Les interventions royales dans les collèges universitaires de Paris, Oxford et Cambridge (XIVe-XVe siècle). Corinne Péneau. Itinéraires du savoir de l'Italie à la Scandinavie (Xe-XVIe siècle). Études offertes à Élisabeth Mornet, Paris, 2009., 25 (1), Publications de la Sorbonne, p. 231-253, 2009, Histoire ancienne et médiévale, 99. hal-00786525

\section{HAL Id: hal-00786525 \\ https://hal.science/hal-00786525}

Submitted on 14 Feb 2013

HAL is a multi-disciplinary open access archive for the deposit and dissemination of scientific research documents, whether they are published or not. The documents may come from teaching and research institutions in France or abroad, or from public or private research centers.
L'archive ouverte pluridisciplinaire HAL, est destinée au dépôt et à la diffusion de documents scientifiques de niveau recherche, publiés ou non, émanant des établissements d'enseignement et de recherche français ou étrangers, des laboratoires publics ou privés. 


\section{Rex Fundator}

\section{Les interventions royales dans les collèges universitaires de Paris, Oxford et Cambridge $\left(\mathrm{XIV}^{\mathrm{e}}-\mathrm{Xv}^{\mathrm{e}}\right.$ siècle)}

Si les études ne manquent pas sur les relations qu'entretiennent les universités avec les pouvoirs princiers à la fin du Moyen Âge, rares sont celles qui s'attachent à ces institutions universitaires particulières que sont les collèges séculiers ${ }^{1}$. Il est vrai que ces établissements destinés à accueillir des étudiants pauvres ne concernaient qu'une partie du monde universitaire médiéval, dans la mesure où, même dans les cités pourvues de nombreux collèges, les boursiers n'ont jamais représenté qu'une minorité d'étudiants ${ }^{2}$. Trois universités cependant se sont nettement détachées des autres par la précocité et la densité de leur infrastructure collégiale : il s'agit de Paris, d'Oxford et de Cambridge. Si l'ancienneté et l'importance des collèges anglais restent connues de tous, il faut toutefois rappeler que Paris fut le véritable berceau du système collégial, the true home of the collegiate system, selon l'expression d'Hastings Rashdall $^{3}$. On y fonde, dès 1180 , le collège des Dix-Huit, reconnu comme le premier établissement de ce type en Occident, puis, en 1257, le collège de Sorbonne, dont l'influence s'étendit bien au-delà des frontières du royaume. Le rythme des fondations parisiennes s'accélère par la suite pour atteindre des sommets au XIV ${ }^{\mathrm{e}}$ siècle, où l'on dénombre trente-sept créations, contre dix-huit dans tout le reste du Moyen Âge ${ }^{4}$. Ainsi, à la fin $\mathrm{du} \mathrm{Xv}^{\mathrm{e}}$ siècle, les deux universités anglaises ne totalisaient que vingt-trois collèges séculiers, alors que la ville de Paris comptait encore, à elle seule, plus de quarante institutions, de taille certes plus modeste. Mais, à côté du système collégial, les universités de Paris, d'Oxford et de Cambridge partageaient d'autres traits communs.

Il s'agissait tout d'abord de trois institutions organisées sur le même modèle, Oxford et Cambridge ayant fidèlement adapté la structure parisienne de l'université de maîtres, par opposition aux universités d'étudiants de type bolonais. Le rôle des collèges au sein de ces organisations hiérarchisées posait donc le même type de problèmes de part et d'autre de la Manche. Ces universités étaient, en outre, les principaux centres d'études de leurs royaumes respectifs. En plus d'être le studium de la capitale, Paris était la plus ancienne et la plus prestigieuse université française. Quant à Oxford et Cambridge, elles furent même les seules universités d'Angleterre durant tout le Moyen Âge ${ }^{5}$. Cette situation de monopole explique qu'elles aient, toutes les trois, attiré l'attention de souverains centralisateurs comme les rois de France et d'Angleterre. Enfin, ces trois universités sont nées de manière spontanée à la charnière des $\mathrm{XII}^{\mathrm{e}}$ et XIII ${ }^{\mathrm{e}}$ siècles. En effet, si le pouvoir royal ne fut pas étranger à l'émergence

\footnotetext{
${ }^{1}$ On notera toutefois deux synthèses: A. B. Cobban, « The Role of Colleges in the Medieval Universities of Northern Europe, with Special Reference to England and France », Bulletin of the John Rylands University Library of Manchester, 71 (1989), p. 49-70 ; et N. Gorochov, « Charles V et les collèges parisiens. L'affirmation d'une politique universitaire royale (1364-1380) », dans Paris et ses campagnes sous l'Ancien Régime. Mélanges offerts à Jean Jacquart, M. Balard, J.-Cl. Hervé et N. Lemaître éd., Paris, 1994, p. 187-194.

${ }^{2} \mathrm{Si}$ les boursiers de collèges ont pu atteindre $17 \%$ de la population étudiante à Paris, on estime à un peu plus de $10 \%$ leur proportion parmi les étudiants d'Oxford au XV ${ }^{\mathrm{e}} \mathrm{s}$. (Fr. J. Pegues, « Philanthropy and the Universities in France and England in the Later Middle Ages », dans The Economic and Material Frame of the Mediaeval University, A. L. Gabriel éd., Notre Dame, 1977, p. 75).

${ }^{3}$ H. Rashdall, The Universities of Europe in the Middle Ages, I, Oxford, 1936, p. 498.

${ }^{4}$ Ibid., I, p. 536-539, complété par A. L. Gabriel, The College System in the Fourteenth-Century Universities, Baltimore - Notre Dame, 1962, p. 32-33, n. 26.

${ }^{5}$ Les fondations des universités de St Andrews (1413), Glasgow (1451) et Aberdeen (1494), qui étaient de toute façon étrangères au royaume d'Angleterre, ont finalement peu pesé sur le recrutement d'Oxford et de Cambridge.
} 
de ces corporations scolaires, il ne fut ni le seul, ni le principal acteur de leurs fondations, ce qui faisait du lien entre le roi et l'université une relation à construire. Parallèlement, les monarchies française et anglaise n'ont cessé d'alterner, tout au long de la période, conflits militaires et alliances matrimoniales, ce qui n'a pas manqué de susciter une certaine émulation entre ces deux couronnes. Il n'est donc pas inutile d'étudier les liens tissés entre le pouvoir royal et les collèges dans les trois universités qui ont façonné, en France comme en Angleterre, le modèle collégial à la fin du Moyen Âge. S'il convient d'aborder, dans un premier temps, la place des fondations royales dans leur environnement universitaire, cette analyse devra toutefois s'étendre aux autres interventions dans les collèges séculiers, afin de mieux comprendre, en dernier lieu, le rôle de ces établissements dans la relation qu'entretenaient le roi et l'université.

\section{La place des collèges royaux au sein des universités}

Les interventions royales, de loin, les plus visibles sont celles qui consistent à créer, de toutes pièces, un collège universitaire. Ce genre d'initiatives était courant dans l'Empire, où le prince était aussi le principal fondateur du studium ${ }^{6}$. On ne compte, en revanche, que quatre fondations entièrement et exclusivement royales sur l'ensemble des universités françaises et anglaises : deux se trouvent à Paris, les deux autres à Cambridge.

Le tout premier collège royal fut fondé à Paris, en mars 1305. Peu de temps avant sa mort, la reine Jeanne de Navarre ( $† 1305)$, épouse de Philippe IV le Bel (1285-1314), créa en effet un établissement de soixante-dix boursiers en grammaire, arts et théologie, qui devaient être choisis dans l'ensemble du royaume ${ }^{7}$. Les bourses du collège de Navarre étaient payées par une rente annuelle de deux mille livres, prélevée sur la recette royale du comté de Champagne $^{8}$. La construction des bâtiments, achevée en 1315, nécessita l'expropriation de nombreux résidants sur la Montagne Sainte-Geneviève et fut entièrement financée par le Trésor ${ }^{9}$. Personne ne contestait, à l'époque, le caractère royal de cette institution. Quelques années plus tard, en mai 1325, la veuve de Philippe V le Long († 1322), Jeanne de Bourgogne $(† 1330)$, fonda à son tour un établissement à Paris : le collège de Bourgogne était destiné à accueillir vingt boursiers en arts, originaires de Franche-Comté ${ }^{10}$. Sa dotation reposait sur la vente du tristement célèbre hôtel de Nesle, propriété de la fondatrice. Mais, une fois acheté le bâtiment du collège, il ne restait plus, pour entretenir les boursiers, qu'une rente annuelle de deux cents livres, soit le dixième des revenus du collège de Navarre ${ }^{11}$. Cette dernière information montre l'écart qui pouvait exister entre les deux institutions, le collège de Bourgogne restant, jusqu'à la fin du Moyen Âge, un établissement relativement modeste. Il

\footnotetext{
${ }^{6}$ Il suffit d'évoquer le Collegium Carolinum (1366) de Prague et le Collegium ducale (1384) de Vienne, respectivement fondés par l'empereur Charles IV et le duc Albrecht II d'Autriche (W. E. Wagner, Universitätsstift und Kollegium in Prag, Wien und Heidelberg. Eine vergleichende Untersuchung spätmittelalterlicher Stiftungen im Spannungsfeld von Herrschaft und Genossenschaft, Berlin, 1999, p. 44-46, 106-110).

${ }^{7}$ Cf. N. Gorochov, Le collège de Navarre de sa fondation (1305) au début du XV siècle (1418). Histoire de l'institution, de sa vie intellectuelle et de son recrutement, Paris, 1997.

${ }^{8}$ Ibid., p. 131-132.

${ }^{9}$ Ibid., p. 154.

${ }^{10}$ P. A. Ford, "John de Martigny, Principal and Benefactor of the College of Burgundy», dans Studium generale. Studies offered to Astrik L. Gabriel..., L. S. Domonkos et R. J. Schneider éd., Notre Dame, 1967, p. 37-38.

${ }^{11}$ L'hôtel de Nesle fut acquis en nov. 1330 par le roi Philippe VI pour une somme de 1000 l. p., augmentée d'une rente annuelle de 200 l. p. payée par le Trésor. Or, les exécuteurs testamentaires de Jeanne de Bourgogne utilisèrent les 1000 1. p. pour acheter le bâtiment du collège (Ford, « John de Martigny... », loc. cit., p. 38).
} 
convient d'ajouter qu'ayant été fondé par une reine douairière et non par une souveraine en titre, il méritait à peine la qualification de collège royal. Enfin, son attachement franc-comtois ne manqua pas d'engendrer pour lui des complications financières, lorsqu'au $\mathrm{XV}^{\mathrm{e}}$ siècle, le roi de France entra en conflit avec le duc de Bourgogne ${ }^{12}$. Il n'est donc pas étonnant que ce soit l'établissement de Jeanne de Navarre qui ait le plus attiré l'attention des souverains.

Le collège de Navarre bénéficiait certes régulièrement de dons royaux, mais une telle sollicitude ne tarda pas à perturber le fonctionnement normal de l'institution. Si Philippe VI de Valois (1328-1350) pratiquait encore un interventionnisme timide dans les nominations aux bourses, son fils, Jean II le Bon (1350-1364), procéda par voie de fait en imposant ses candidats au grand maître du collège ${ }^{13}$. Charles V (1364-1380) adopta, quant à lui, une politique beaucoup plus ambitieuse : entre 1369 et 1374, plusieurs initiatives indiquent qu'il entendait faire du collège de Navarre le centre d'un véritable culte dynastique. En octobre 1369, l'université décida, à la demande du roi, de faire prononcer le sermon de la Saint-Louis (25 août) dans la chapelle du collège, laquelle fut officiellement placée, en octobre 1373, sous l'invocation de ce saint ${ }^{14}$. L'année suivante, Charles V fit introduire au collège la fête de la Présentation de la Vierge pour mieux en assurer la diffusion dans le nord du royaume ${ }^{15}$. Enfin, en mars 1374, le roi rebaptisa tout bonnement l'établissement en «collège de Champagne » et confia l'exécution de cette ordonnance à son confesseur, qui était devenu entre-temps collateur ordinaire des bourses ${ }^{16}$. La prise de contrôle du collège par les Valois semble ainsi s'inscrire dans un vaste programme de propagande contre les derniers avatars du parti navarrais. Le collège de Navarre, lieu de réflexion politique, avait pu apparaître, aux yeux de Charles $\mathrm{V}$, comme un foyer potentiel de contestation dynastique dans une période particulièrement délicate pour la monarchie ${ }^{17}$. Ce n'était donc pas tant l'établissement scolaire qui était visé par ces interventions que le groupe de pression qu'il aurait pu constituer au sein du studium parisien.

En effet, le collège de Navarre était très tôt parvenu à acquérir une place prépondérante à l'université : dès 1318 , celle-ci décidait d'y faire prononcer ses sermons généraux ${ }^{18}$. À la fin du XIV ${ }^{\mathrm{e}}$ siècle, la prestigieuse institution étendait même son influence sur l'ensemble de la corporation et sur plusieurs collèges du Quartier latin : entre 1380 et 1418, quatre chanceliers de l'université, sept des quelque cinquante recteurs connus et les six procureurs de la nation française étaient issus du collège de Navarre ${ }^{19}$. On trouvait en outre des navarristes à la tête des collèges Mignon (1391-1398), de Dormans-Beauvais (1401-1407), de Boissy (14031405) et de Fortet (1416-1418), ainsi qu'au collège de l'Ave Maria, gouverné à partir de 1386 par le grand maître de Navarre $^{20}$. $\mathrm{Au} \mathrm{XV}^{\mathrm{e}}$ siècle, ce collège royal était encore l'objet d'attentions particulières de la part des souverains : après avoir supervisé la réforme générale de l'université de Paris (1452), Charles VII (1422-1461) confia, en octobre 1459, la réforme

\footnotetext{
${ }^{12}$ En 1469, en pleine guerre entre Louis XI et Charles le Téméraire, le Trésor royal suspendit purement et simplement le paiement des bourses du collège (Ford, « John de Martigny... », loc. cit., p. 45).

${ }^{13}$ Il impose, en particulier, le boursier Simon Pauli contre l'avis du grand maître (Gorochov, Le collège de Navarre..., op. cit., p. 233-235, 310-311).

${ }^{14}$ Gorochov, Le collège de Navarre..., op. cit., p. 328-333.

${ }^{15}$ Ibid., p. 337-339.

${ }^{16}$ Ibid., p. 334-336. C'est sans doute entre 1370 et 1372 que le roi confie à son confesseur la collation des bourses du collège, mais on ne conserve aucune trace écrite de cette réforme.

${ }^{17} \mathrm{X}$. de La Selle, Le service des âmes à la Cour. Confesseurs et aumôniers des rois de France du XIII au $X V^{e}$ siècle, Paris, 1995, p. 200.

${ }^{18}$ Document édité par H. Denifle et É. Châtelain, Chartularium universitatis parisiensis, II, Paris, 1891, p. $226-227, \mathrm{n}^{0} 774$.

${ }^{19}$ Gorochov, Le collège de Navarre..., op. cit., p. 531-536.

${ }^{20}$ Ibid., p. 525-528, 644, 695; Th. Kouamé, Le collège de Dormans-Beauvais à la fin du Moyen Âge. Stratégies politiques et parcours individuels à l'université de Paris (1370-1458), Leiden - Boston, 2005, p. 119, 129-131.
} 
de l'établissement à une commission dominée par des membres du Parlement ${ }^{21}$. Leurs travaux aboutirent à 1'ordonnance du 25 juillet 1464, promulguée par Louis XI (1461-1483), qui renforça le caractère disciplinaire des premiers statuts ${ }^{22}$. En janvier 1475 , le roi intervint à nouveau dans l'organisation de l'institution en attribuant au chapitre cathédral de Paris un droit de présentation à une bourse de grammairien pour l'un des enfants de chœur de NotreDame ${ }^{23}$. Ainsi, le collège de Navarre était non seulement le plus important des établissements parisiens, mais il était surtout celui sur lequel la monarchie française exerçait le contrôle le plus étroit.

De l'autre côté de la Manche, seule l'université de Cambridge bénéficia de fondations royales en bonne et due forme avec le King's Hall (1337) et King's College (1441). Le contraste est saisissant, lorsque l'on met en parallèle la présence de ces deux collèges royaux à Cambrigde et leur absence totale à Oxford, qui demeura pourtant la plus importante université d'Angleterre jusqu'à la Renaissance. Les historiens britanniques attribuent habituellement cette distorsion à une politique délibérée de la Couronne : la réaction contre le wycliffisme oxonien aurait orienté le patronage royal vers Cambridge, considérée comme la nouvelle école de l'Église, capable de supplanter une université d'Oxford qui s'était gravement égarée ${ }^{24}$. Il est certain que les actes délivrés par Henri VI (1422-1461) en faveur des collèges de Cambridge rendent compte d'une telle prévention. Ainsi, le souverain autorise la création du collège Saint-Bernard, ancêtre de Queens' College, parce qu'un tel établissement doit permettre «1'extirpation des hérésies et des erreurs, l'augmentation de la foi, le profit du clergé et la stabilité de l'Église » $(1447)^{25}$. Henri VI relie d'ailleurs la fondation de son propre collège royal au développement des hérésies qui perturbent l'Angleterre $(1443)^{26}$. Enfin, une addition aux statuts de King's College exige que tout nouvel arrivant prête serment de ne pas soutenir les théories de Wyclif ou de Pecock ${ }^{27}$. Mais, si ces dispositions s'expliquent tout à fait dans le contexte du milieu du Xve siècle, elles ne peuvent guère éclairer le choix délidéré de Cambridge, dès le début du XIV siècle, pour la fondation du premier collège royal anglais. Selon Alan Cobban, Édouard II (1307-1327) aurait en fait

\footnotetext{
${ }^{21}$ Document édité par J. de Launoy, Regii Navarrae gymnasii parisiensis historia, I, Paris, 1677, p. 165-168. La commission comprenait le cardinal Richard Olivier de Longueil, conseiller du roi, Hélie de Tourettes, président au Parlement, Jean de Courcelles, Jean de La Réaulté et Jean de Montigny, conseillers au Parlement, Étienne Chevalier et Pierre Beccart, trésoriers de France, Jean Dauvet, procureur général au Parlement, et Guillaume de Chateaufort, grand maître du collège. Mais seuls Hélie de Tourettes († 1461), Jean de Courcelles, Jean de Montigny, Jean Dauvet et Guillaume de Chateaufort prirent réellement part aux travaux.

${ }^{22}$ Document édité par Launoy, Regii Navarrae..., op. cit., I, p. 170-177.

${ }^{23}$ La Selle, Le service des âmes à la Cour..., op. cit., p. 202.

${ }^{24}$ Rashdall, The Universities of Europe..., op. cit., III, p. 316 ; et plus récemment : A. B. Cobban, The Medieval English Universities : Oxford and Cambridge to c. 1500, Berkeley - Los Angeles, 1988, p. 131-133.

${ }^{25}$ J. Twigg, A History of Queens' College, Cambridge, 1448-1986, Woodbridge, 1987, p. 3. On peut noter que la situation paraît beaucoup moins tendue à peine 30 ans plus tard, comme le montre cette disposition des statuts de Queens' College (1475), qui interdit aux boursiers de défendre des propositions hérétiques... sauf pour des nécessités académiques: «Statuimus quod nullus socius hujus collegii serio defendat, neque publice neque privatim, haereses vel errores anabaptistarum et libertinorum, nec quamcunque aliam opinionem contra sacrae Scripturae auctoritatem, nisi tantum eruditionis gratia, et praehabita protestatione » (A. L. Gabriel, « Motivation of the Founders at Mediaeval Colleges », dans Garlandia. Studies in the History of the Mediaeval University, Notre Dame - Frankfurt-am-Main, 1969, p. 223, n. 42).

${ }^{26}$ Dans ses lettres patentes du 10 juil. 1443, le roi explique qu'il a fondé King's College «ad honorem omnipotentis Dei [...] extirpacionem quoque heresum et errorum qui solemnium regnorum ac universitatum pacem perturbant regnumque nostrum Angliae »(A. B. Cobban, The King's Hall within the University of Cambridge in the Later Middle Ages, Cambridge, 1969, p. 26, n. 1).

${ }^{27}$ Cette addition aux statuts de 1453 fut sans doute insérée entre 1457 et 1459 (J. Saltmarsh, « King's College », dans A History of the County of Cambridge and the Isle of Ely, J. P. C. Roach éd., III, The City and University of Cambridge, London, 1959, p. 382).
} 
souhaité rétablir son prestige personnel à l'intérieur du royaume en favorisant une université plus strictement insulaire ${ }^{28}$. Quelles que fussent les raisons de cette faveur royale, il n'en reste pas moins que les souverains anglais firent de la modeste université de Cambridge le champ d'expérimentation de leur politique universitaire.

La préhistoire du King's Hall est, de ce point de vue, tout aussi intéressante que son histoire. C'est en juillet 1317 qu'est mentionné pour la première fois, dans un writ d'Édouard II, l'entretien d'un clerc et de douze enfants de la Chapelle royale étudiant ensemble à Cambridge aux frais du Trésor ${ }^{29}$. À peine deux ans plus tard, cette communauté compte déjà trente-trois membres, selon un compte de l'Échiquier (1319) ${ }^{30}$. Or, l'installation de ces écoliers du roi correspond exactement au moment où le souverain cherche à faire reconnaître par le pape le statut de studium generale pour l'université de Cambridge ${ }^{31}$. Même si la paternité du premier collège royal anglais revient sans conteste à Édouard II, c'est néanmoins son fils, Édouard III (1327-1377), qui fonda officiellement le King's Hall en octobre 1337 : il dote alors l'institution de revenus stables et de bâtiments capables de loger ses trente-deux boursiers ${ }^{32}$. Mais ce n'est qu'en 1380 que de nouveaux statuts royaux reconnaissent à cette communauté un certain droit de regard sur le gouvernement du collège, comme c'était le cas dans la plupart des autres établissements d'Angleterre ${ }^{33}$. Le fait est que le King's Hall resta longtemps une exception parmi les collèges anglais. Avant 1337, cette institution hybride n'était ni un collège, doté de revenus propres, ni un hostel, offrant un hébergement payant, dans la mesure où ses boursiers étaient directement entretenus par le Trésor $^{34}$. Jusqu'au début du $\mathrm{XV}^{\mathrm{e}}$ siècle, elle fonctionnait en outre comme une sorte d'extension universitaire de la Cour ou, plus exactement, de la Chapelle royale, dont les membres pouvaient ainsi parfaire leur formation juridique ${ }^{35}$. Enfin, cet établissement resta sous le contrôle direct de la Couronne jusqu'à sa disparition dans Trinity College en 1546: le roi nommait personnellement chacun des boursiers et bien entendu le maître, qui était responsable de son office devant lui seul ${ }^{36}$. Ainsi, dès le début du $\mathrm{XIV}^{\mathrm{e}}$ siècle, le fonctionnement même du King's Hall révélait un lien fort entre l'université de Cambridge et la monarchie.

Au milieu du $\mathrm{XV}^{\mathrm{e}}$ siècle, dans un tout autre contexte, Henri VI renoua avec cette politique en créant son prestigieux King's College, le 12 février $1441^{37}$. Cette nouvelle fondation était réservée à soixante-dix boursiers en arts, droit et théologie, issus du collège royal d'Eton, qui venait d'être établi, en octobre 1440 , près du château de Windsor ${ }^{38}$. Sa dotation, d'un revenu annuel de mille livres, reposait sur les nombreux biens confisqués par le roi, grâce à la suppression des prieurés étrangers ${ }^{39}$. Un vaste quartier délabré, situé entre la High Street et la rivière Cam, fut rasé pour permettre de construire un imposant édifice et une monumentale chapelle, suivant les plans du souverain, mais la guerre des Deux-Roses suspendit les

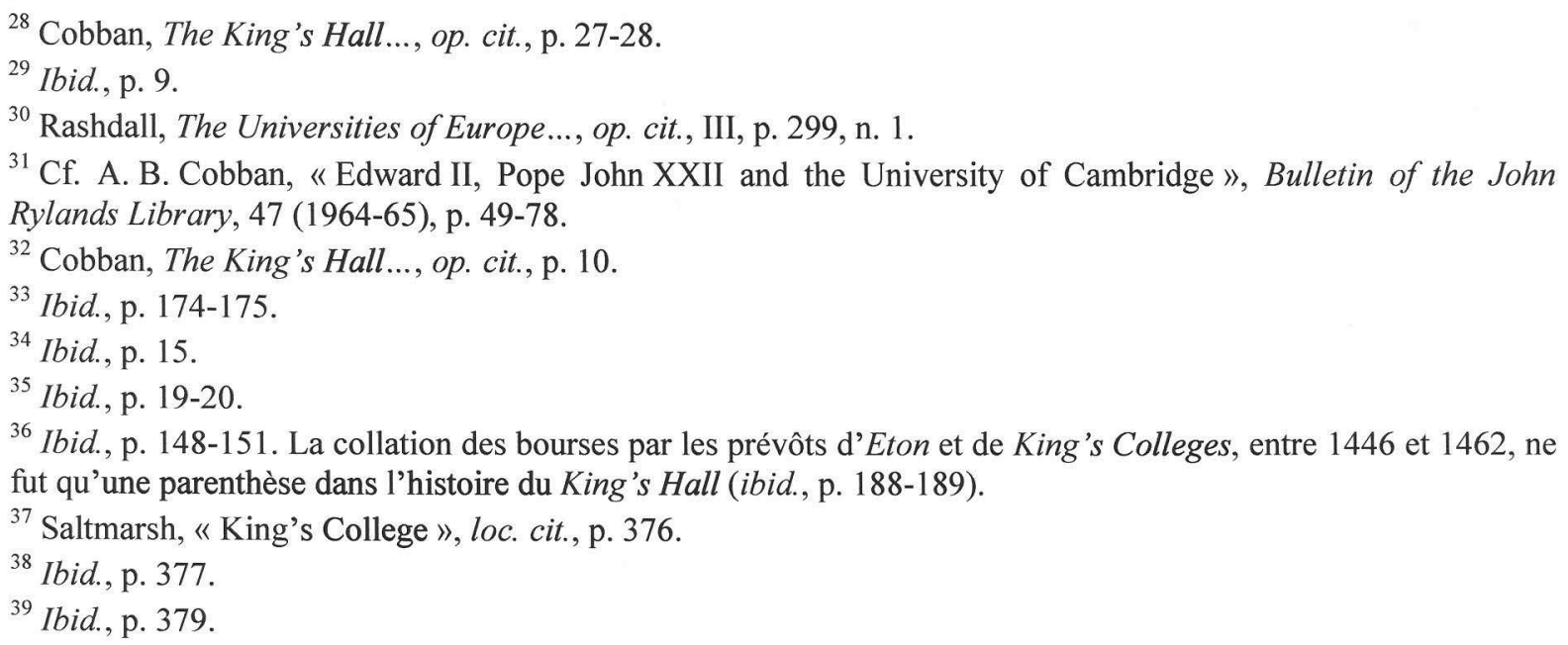


travaux ${ }^{40}$. Si le projet d'Henri VI fut sans doute suscité, sinon encouragé par John Langton, alors chancelier de l'université de Cambridge et maître de Pembroke College ${ }^{41}$, il est difficile de ne pas voir dans cette fondation la transposition pure et simple du non moins célèbre New College d'Oxford, créé par William of Wykeham en lien étroit avec son collège de Winchester (1379). Au-delà de la simple reproduction du modèle institutionnel, le choix des hommes suggérait déjà des rapprochements. Henri VI prit soin, en effet, de nommer l'ancien maître de Winchester College à la tête de son collège d'Eton ${ }^{42}$. Surtout, ces quatre institutions conclurent, dès juillet 1444, une Amicabilis Concordia, montrant clairement que le roi cherchait, par tous les moyens, à rapprocher sa fondation de celle de Wykeham ${ }^{43}$.

Par la volonté d'Henri VI, King's College acquit rapidement une place prépondérante au sein du studium. En plus de ses soixante-dix boursiers, l'établissement entretenait déjà dix chapelains, six clercs et seize enfants de chœur ${ }^{44}$. Entre 1444 et 1446, Henri VI lui accorda, en outre, un grand nombre de privilèges royaux et obtint du pape qu'il fût exempté des juridictions de l'archevêque de Cantorbéry, de l'évêque d'Ely et surtout du chancelier de Cambridge, au profit du seul évêque de Lincoln (1445), faisant ainsi du collège une véritable enclave judiciaire au sein des circonscriptions dont il aurait dû dépendre ${ }^{45}$. Or, à cette date, l'université de Cambridge avait déjà été soustraite aux tutelles ecclésiastiques d'Ely (1401) et de Cantorbéry $(1433)^{46}$. La réelle nouveauté de la bulle d'exemption résidait donc dans l'indépendance de King's College vis-à-vis des autorités universitaires. Si la corporation fit d'abord mine d'accepter une telle dérogation à ses propres privilèges (1448), elle revint plus tard sur sa décision en refusant d'admettre aux grades les Kingsmen qui ne se seraient pas soumis à l'autorité de son chancelier (1454). Après trois ans de conflit, les deux parties finirent, malgré tout, par trouver un accord, l'université acceptant que le collège exerçât une juridiction sur ses propres membres pour les actes commis à l'intérieur de ses locaux $(1457)^{47}$. Paradoxalement, cette faveur royale fut à l'origine de graves difficultés. Considéré par les yorkistes comme le symbole du pouvoir lancastrien, King's College connut une situation matérielle catastrophique sous le règne d'Édouard IV (1461-1483), qui n'hésita pas à dénoncer une grande partie des donations faites par son prédécesseur ${ }^{48}$. La santé financière de l'institution resta donc précaire jusqu'au milieu du XVI siècle.

La comparaison entre les collèges royaux fondés à Paris et à Cambridge appelle quelques commentaires. En terme d'effectifs, les fondations royales offrent un nombre de bourses équivalent de part et d'autre de la Manche: les collèges de Navarre et de Bourgogne accueillent ensemble quatre-vingt-dix étudiants, tandis que le King's Hall et King's College en entretiennent une centaine à eux deux. Mais, si l'on rapporte ces chiffres à la population totale des universités, force est de constater que les boursiers royaux sont proportionnellement deux fois et demi plus nombreux à Cambridge $(7,8 \%)$ qu'à Paris $(3 \%)^{49}$. Une autre

\footnotetext{
${ }^{40}$ A History of the University of Cambridge, Chr. Brooke dir., I, D. R. Leader, The University to 1546, Cambridge, 1988 , p. 228, 230-231.

${ }^{41}$ Saltmarsh, « King's College », loc. cit., p. 377.

${ }^{42}$ A. B. Cobban, "Colleges and Halls, 1380-1500», dans The History of the University of Oxford, II, Late Medieval Oxford, J. I. Catto et T. A. R. Evans éd., Oxford, 1992, p. 610. Ce dernier n'est autre que William Waynflete, évêque de Winchester (1447), fondateur de Magdalen Hall (1448) et chancelier d'Angleterre (1456).

${ }^{43}$ Saltmarsh, «King's College», loc. cit., p. 377. L'Amicabilis Concordia était une alliance perpétuelle entre Eton, King's, Winchester et New Colleges, qui s'assuraient, par là même, une entraide judiciaire.

${ }^{44}$ Saltmarsh, « King's College », loc. cit., p. 382.

${ }^{45}$ Ibid., p. 377.

${ }^{46}$ Cobban, The Medieval English Universities..., op. cit., p. 289, 294.

${ }^{47}$ Saltmarsh, « King's College », loc. cit., p. 378.

${ }^{48}$ Ibid., p. 379.

${ }^{49} \mathrm{Si}$ l'on considère qu'au milieu du XV ${ }^{\mathrm{e}}$ s., Cambridge comptait environ 1300 étudiants et Paris environ 3000.
} 
différence essentielle distingue les fondateurs de ces institutions : en France, il ne s'agit que de reines, tandis qu'en Angleterre ce sont d'abord des rois. Il semble qu'au début du $X I V{ }^{\mathrm{e}}$ siècle, les souverains français aient encore considéré les collèges séculiers comme de simples institutions charitables, ce qu'ils étaient, du reste, assurément à cette date, alors que les rois d'Angleterre avaient déjà compris l'intérêt potentiel de ces établissements pour la formation de leurs élites administratives. Le type de bourses offertes dans ces collèges illustre parfaitement ce décalage : les statuts de Navarre et de Bourgogne ne font que confirmer la place prépondérante des théologiens et des artiens à l'intérieur du studium parisien, tandis que le King's Hall n'est destiné, dès l'origine, qu'à des juristes issus de l'entourage du roi. Audelà de ces différences, on peut toutefois noter quelques points communs. Soucieux de conserver un contrôle étroit sur leur fondation, Édouard II et Édouard III se sont incontestablement inspirés des statuts des collèges parisiens pour formaliser la mise sous tutelle extérieure du King's Hall, en rupture avec la tradition d'autogestion qui se pratiquait, jusqu'alors, dans les établissements anglais ${ }^{50}$. Surtout, les souverains des deux pays ont tenu à faire de l'une de ces fondations scolaires la manifestation universitaire de leur majesté royale. Ainsi, Navarre et King 's College comptaient parmi les plus prestigieux collèges d'Occident, à l'instar de la Sorbonne (1257), de Merton (1264), du Carolinum (1366) de Prague ou de New College (1379) à Oxford.

\section{Les interventions royales dans les collèges séculiers}

On ne s'est intéressé, jusqu'à présent, qu'aux collèges directement fondés par des souverains. Mais, aussi prestigieuses soient-elles, ces institutions ne résument pas, à elles seules, la politique royale à l'égard des collèges. Les rois ne se sont pas privés, en effet, d'intervenir personnellement dans des établissements qui n'avaient pas été fondés par eux, mais par des membres de leur entourage.

L'historiographie a souvent insisté, à juste titre, sur le grand nombre de prélats qui avaient créé des collèges. Mais cela ne doit pas faire oublier le poids des officiers du roi, même si ceux-ci ont pu accomplir, par ailleurs, de plus belles carrières dans l'Église. On constate en effet qu'environ un tiers des fondateurs étaient issus de la Cour ou servaient directement le souverain. Ainsi, sur les cinquante-cinq institutions fondées à Paris au Moyen Âge, seize le furent par des collaborateurs ou des serviteurs du roi de France : neuf avaient exercé ou exerçaient encore des fonctions politiques ${ }^{51}$, cinq appartenaient à l'Hôtel ${ }^{52}$ et deux à la haute

\footnotetext{
${ }^{50}$ Cobban, The King's Hall..., op. cit., p. 151.

${ }^{51}$ On trouve un chancelier de France, Jean de Dormans, fondateur du collège de Dormans-Beauvais (1370), un ancien garde des sceaux, Gilles Aycelin de Montaigu, fondateur du collège de Montaigu (1314), un président de la Chambre des comptes, Hugues d'Arcy, cofondateur du collège de Cambrai (1348), trois anciens maîtres des requêtes de l'Hôtel, André Ghini, cofondateur du collège des Lombards (1334), Pierre Bertrand, fondateur du collège d'Autun (1341), et Grégoire Langlois, fondateur du collège de Sées (1404), un secrétaire du roi, Geoffroy du Plessis, fondateur du collège du Plessis (1322), et de proches conseillers du roi, Raoul et Robert d'Harcourt, fondateurs du collège d'Harcourt (1280), ainsi que Raoul de Presles, fondateur du collège de Presles (1324).

${ }^{52}$ On trouve un chapelain du roi, Robert de Sorbon, fondateur de la Sorbonne (1257), un trésorier de la SainteChapelle du Palais, Guy de Laon, fondateur du collège de Laon (1324), deux médecins du roi, Gervais Chrétien, fondateur du collège de Maître Gervais (1371), et Jean de Guistry, refondateur du collège de Cornouaille (1379), ainsi qu'un maître d'hôtel, Jean de Dainville, cofondateur du collège de Dainville (1380). Ajoutons que François de l'Hôpital et Renier Jean, cofondateurs du collège des Lombards (1334), évoqué dans la note précédente, étaient respectivement clerc des albalétriers du roi et apothicaire de la reine Jeanne de Bourgogne.
} 
administration $^{53}$. Ces seize institutions délivraient, en outre, quelque deux cent cinquante bourses d'écoliers, ce qui pouvait considérablement élargir le champ d'action de la politique royale $^{54}$. De l'autre côté de la Manche, sur les vingt-trois collèges séculiers fondés à Oxford et Cambridge, neuf le furent par des officiers du roi d'Angleterre. Nettement moins nombreux qu'à Paris, ces établissements parvenaient toutefois à offrir un nombre équivalent de bourses $^{55}$. On trouvait aussi, parmi leurs fondateurs, sept membres du gouvernement, dont quatre chanceliers d'Angleterre ${ }^{56}$. Quant à leur implantation géographique, elle était exactement inverse à celle des collèges royaux, puisque sept d'entre eux se trouvaient à Oxford et deux à Cambridge ${ }^{57}$. L'existence d'une telle quantité de collèges séculiers créés dans l'entourage des rois explique, en grande partie, les interventions de ces derniers en dehors des fondations royales. C'est souvent, en effet, pour récompenser un proche serviteur ou pour exaucer ses dernières volontés que les souverains ont interféré dans la vie de ces établissements. On peut ainsi répartir les interventions royales en trois catégories: les libéralités, les confirmations et les refondations.

Les rois de France, depuis saint Louis (1226-1270), ont toujours prêté attention aux fondations universitaires, comme à toute œuvre pie des membres de la Cour. On connaît, en effet, la faveur du roi pour le collège de son chapelain, Robert de Sorbon ${ }^{58}$. Quant à Philippe VI, il faisait régulièrement verser par le Trésor des sommes importantes à plusieurs établissements parisiens, parmi lesquels les collèges de Bayeux, des Cholets et du Cardinal Lemoine $^{59}$. Ces libéralités pouvaient aussi prendre la forme de dotations foncières. C'est le cas à Oxford, en 1456, lorsque Henri VI fait don de l'hôpital Saint-Jean-Baptiste à son chancelier, William Waynflete, pour lui permettre d'y construire un St Magdalen Hall, qui

\footnotetext{
${ }^{53}$ Jean de Huban, fondateur du collège de l'Ave Maria (1336), était président à la chambre des enquêtes du Parlement, tandis que Jean Mignon, fondateur du collège Mignon (1343), était maître clerc à la Chambre des comptes.

${ }^{54}$ On obtient un total théorique de 250 bourses, si l'on additionne les 40 boursiers du collège d'Harcourt en 1311 (M.-M. Compère, Les collèges français, $16^{e}-18^{e}$ siècles..., III, Paris, 2002, p. 178), les 16 du collège de Laon et les 13 du collège de Presles en 1324 (ibid., p. 201, 317), les 19 de la Sorbonne en 1329-1330 (W. J. Courtenay, Parisian Scholars in the Early Fourteenth Century..., Cambridge, 1999, p. 221), les 25 du collège du Plessis en 1332 (Compère, Les collèges français..., op. cit., III, p. 305), les 11 du collège des Lombards (ibid., p. 222), les 6 du collège de l'Ave Maria (ibid., p. 85), les 15 du collège d'Autun (ibid., p. 80), les 12 du collège Mignon (ibid., p. 260), les 19 du collège de Cambrai (ibid., p. 128), les 24 du collège de Dormans-Beauvais (ibid., p. 98), les 24 du collège de Maître Gervais (ibid., p. 241), les 10 du collège de Cornouaille en 1380 (ibid., p. 149), les 10 du collège de Dainville (ibid., p. 152) et les 6 du collège de Sées en 1428 (ibid., p. 326). Les bourses du collège de Montaigu ne figurent pas dans cette liste, car il ne reste aucune trace de l'exécution de la fondation initiale, au début du XIV ${ }^{\mathrm{e}}$ s. (ibid., p. 268).

${ }^{55}$ On obtient un total théorique de 246 bourses, si l'on additionne les 20 boursiers de Merton College (Rashdall, The Universities of Europe..., op. cit., III, p. 193), les 12 d'Exeter College (ibid., p. 202), les 6 de Michaelhouse (ibid., p. 301), les 10 d'Oriel College en 1326 (ibid., p. 205), les 12 de Queen's College (ibid., p. 208), les 70 de New College (ibid., p. 215), les 40 d'All Souls College (ibid., p. 227), les 40 fellows et 30 demies de Magdalen College en 1458 (ibid., p. 230), ainsi que les 6 boursiers de Jesus College (ibid., p. 496).

${ }^{56}$ Les quatre chanceliers d'Angleterre sont Walter of Merton, fondateur de Merton College (1264), William of Wykeham, fondateur de New College (1379), William Waynflete, fondateur de Magdalen College (1448), et John Alcock, fondateur de Jesus College (1496). Les autres collaborateurs du souverain sont Walter Stapeldon, fondateur d'Exeter College (1314), qui devint trésorier d'Angleterre, Hervey of Stanton, qui fonda Michaelhouse (1324), lorsqu'il était chancelier de l'Échiquier, et Henry Chichele, cofondateur d'All Souls College (1438) et principal membre du Conseil. On note enfin la présence de deux officiers de l'Hôtel : Adam of Brome, clerc de la chancellerie et aumônier du roi, qui fonda Oriel College (1324), et Robert of Eglesfield, chapelain de la reine et cofondateur de Queen's College (1341).

${ }^{57}$ Merton, Exeter, Oriel, Queen's et New Colleges, ainsi qu'All Souls et Magdalen Colleges ont été fondés à Oxford, alors que Michaelhouse et Jesus College l'ont été à Cambridge.

${ }^{58}$ P. Glorieux, Aux origines de la Sorbonne, I, Paris, 1966, p. 88-91.

${ }^{59}$ Gorochov, Le collège de Navarre..., op. cit., p. 224.
} 
devait devenir Magdalen College $(1458)^{60}$. À côté de ces dons directs, le roi de France pouvait aussi offrir l'amortissement des biens de la dotation: cela consistait à exempter le fondateur du versement d'un droit que le Trésor avait 1'habitude de prélever sur les biens tombant en mainmorte. C'est ainsi qu'entre 1368 et 1373, Charles V amortit gracieusement toutes les rentes que son chancelier, Jean de Dormans, était en train d'acheter pour doter son collège $^{61}$. Dans le système anglais de monarchie féodale, l'autorisation du roi était même un préalable indispensable à la constitution de toute dotation. Le fondateur d'un collège devait en fait acheter une licence royale, qui lui donnait le droit de tenir un domaine acquis en mainmorte, approuvait son intention de créer une nouvelle communauté et permettait à d'autres donateurs d'augmenter la future fondation, qui se voyait ainsi reconnaître une personnalité juridique ${ }^{62}$. La délivrance gracieuse de telles licences faisait aussi partie des faveurs que les rois d'Angleterre pouvaient réserver aux fondations universitaires.

À cette autorisation préalable répondait, en France comme en Angleterre, la possibilité de confirmer, a posteriori, les fondations et les statuts de collèges. Un tel acte ne pouvait toutefois pas être assimilé aux licences royales anglaises, dans la mesure où la confirmation n'était ni systématique, ni même nécessaire. Il s'agissait bien d'une faveur faite au fondateur, puisqu'en acceptant de ratifier telle ou telle fondation, le souverain délivrait des instruments publics d'une valeur autrement plus grande que les actes notariés. C'est ainsi que Guy de Laon et Raoul de Presles, tous deux serviteurs du roi, obtinrent de Philippe le Bel qu'il confirmât la fondation de leur collège en janvier $1314^{63}$. Cette confirmation royale tenait d'ailleurs lieu de charte de fondation, car les archives des collèges de Presles et de Laon n'ont conservé aucun acte antérieur. La ratification était toutefois une arme à double tranchant : en étendant la protection du roi sur la fondation, elle constituait aussi un puissant levier d'intervention pour l'administration royale et pouvait, à moyen terme, remettre en question l'indépendance même de l'institution. Les déboires de Lincoln College, à Oxford, en sont une bonne illustration. Après la chute d'Henri VI, cet établissement aux sympathies lancastriennes jugea plus prudent d'obtenir d'Édouard IV la confirmation de sa charte de fondation, qui datait de 1429. Le roi finit par accéder à cette demande en janvier 1463, mais l'omission, dans le nouvel acte, de l'expression et successoribus, après les mots rectori et scholaribus, pour désigner les détenteurs du patrimoine du collège, fit craindre que leurs biens ne tombassent en déshérence, après la mort du maître et des derniers boursiers présents lors de la ratification. Or, il fallut attendre l'année 1478 pour que cette incertitude sur la personnalité juridique de l'institution fût définitivement levée avec la rédaction d'une seconde charte de confirmation ${ }^{64}$. Cet exemple montre à quel point le roi pouvait interférer dans la vie d'un établissement. Mais l'intervention royale la plus radicale consistait encore, pour le souverain, à se substituer purement et simplement au fondateur initial.

Le premier cas de refondation est anglais et s'inscrit dans la politique universitaire d'Édouard II, qui fut, comme on sait, à l'origine du premier collège royal de Cambridge. Le 21 janvier 1326, le roi reprit à son compte la fondation du collège Sainte-Marie d'Oxford, plus connu sous le nom d'Oriel College, qui avait été créé deux ans plus tôt par son aumônier

\footnotetext{
${ }^{60}$ Rashdall, The Universities of Europe..., op. cit., III, p. 229.

${ }^{61}$ Kouamé, Le collège de Dormans-Beauvais..., op. cit., p. 46, 49.

${ }^{62}$ E. F. Jacob, «Founders and Foundations in the Later Middle Ages », dans Essays in Later Medieval History, Manchester - New York, 1968, p. 155.

${ }^{63}$ Document édité par C. Fabris, Étudier et vivre à Paris au Moyen Âge. Le collège de Laon (XIV $V^{e} X V^{e}$ siècles), Paris, 2005, p. 311-313, n 1 . Ce collège de Laon-Presles finit par être séparé en deux établissements au début de l'année 1324 (ibid., p. 44).

${ }^{64}$ V. Green, The Commonwealth of Lincoln College, 1427-1977, Oxford, 1979, p. 36-38.
} 
Adam of Brome $(\uparrow 1332)^{65}$. Édouard II ne se contentait pas d'une simple confirmation; il changeait le contenu même de la première fondation. Alors qu'Adam of Brome avait prévu dix boursiers en théologie, les statuts royaux permettaient que cinq ou six d'entre eux pussent étudier le droit canonique et même le droit civil. Surtout, le roi confiait à son chancelier le soin d'intervenir au collège à l'occasion de l'élection du prévôt ${ }^{66}$. Cette refondation royale ressemblait toutefois plus à une "cofondation », compte tenu de la collaboration active du fondateur initial, qui avait pris soin de céder tous les biens de son collège à la Couronne ${ }^{67}$. Quoi qu'il en soit, à la fin de son règne, Édouard II contrôlait plus ou moins directement un collège séculier dans chacune des deux universités de son royaume. Si son fils, Édouard III, abandonna son droit de visite au profit de l'évêque de Lincoln, il ne se désintéressa pas pour autant de cette cofondation royale : il la dota de plusieurs biens fonciers et immobiliers, parmi lesquels un bâtiment dénommé « le Oriole » (1327), qui finit par donner son nom à toute l'institution ${ }^{68}$. Comme pour le King's Hall, la politique universitaire d'Édouard III aura donc consisté à consolider les institutions mises en place par son père. Une opération similaire eut lieu quinze ans plus tard avec un autre collège d'Oxford, à la différence près que ce ne fut pas le roi, mais la reine qui y fut associée. En janvier 1341, Robert of Eglesfield († 1349), chapelain de Philippa de Hainaut $(\uparrow 1369)$, fondait Queen's College, en hommage à sa maîtresse, et plaçait son institution sous le patronage perpétuel des reines d'Angleterre ${ }^{69}$. Ce n'était donc pas seulement l'épouse d'Édouard III qui était honorée, mais bel et bien la Couronne, même si Philippa de Hainaut se considéra, dès l'origine, comme la principale instigatrice de cette cofondation ${ }^{70}$. Robert of Eglesfield suivait ainsi l'exemple d'Adam of Brome et nourrissait sans doute aussi l'espoir d'attirer la bienveillance royale sur sa communauté. La faveur de la reine entraîna en effet celle du roi, qui donna à Queen's College de nombreux revenus ecclésiastiques et fit de cet établissement l'un des mieux dotés d'Oxford au milieu du XIV ${ }^{\mathrm{e}}$ siècle $^{71}$.

Il n'est pas impossible que ces interventions anglaises aient influencé, de l'autre côté de la Manche, la politique des rois de France, qui s'étaient jusqu'alors bien gardés de fonder des collèges, laissant aux reines le soin d'accomplir ce devoir charitable. L'initiative en revient à Jean le Bon, qui profita de la déshérence d'une fondation voulue par l'un de ses officiers, Jean Mignon († 1343). Ce dernier exprimait, dans son testament, la volonté de fonder un collège de douze boursiers choisis parmi les membres de sa famille (1343). Or, dix ans plus tard, son frère Robert, principal exécuteur de la fondation, n'avait toujours pas installé les boursiers. En juillet 1353 , le roi ordonna donc la fondation du collège et s'attribua, en tant que nouveau

\footnotetext{
${ }^{65}$ La charte de refondation et les statuts royaux sont datés du même jour (C. L. Shadwell et H. E. Salter, Oriel College Records, Oxford, 1926, p. 3-5, n 3 ; p. 5-14, n 4).

${ }^{66}$ Shadwell et Salter, Oriel College Records, op. cit., p. 6-7. Cette disposition n'est cependant pas reprise dans les statuts que se donne le collège le 23 mai 1326 (Rashdall, The Universities of Europe..., op. cit., III, p. 204-205)

${ }^{67}$ J. R. L. Highfield, « The Early Colleges », dans The History of the University of Oxford, I, The Early Oxford Schools, J. I. Catto et T. A. R. Evans éd., Oxford, 1984, p. 237.

${ }^{68}$ T. H. Aston et R. Faith, « The Endowments of the University and Colleges to circa 1348 », dans The History of the University of Oxford, op. cit., I, p. 302-303. En ce qui concerne « le Oriole », Édouard III donna en fait au collège son droit de réversion sur une maison qui aurait dû revenir à la Couronne, après la mort de son possesseur (Shadwell et Salter, Oriel College Records, op. cit., p. 118-119, n 101).

${ }^{69}$ R. H. Hodgkin, «The Queen's College », dans The Victoria History of the County of Oxford, H. E. Salter et M. D. Lobel éd., III, The University of Oxford, London, 1954, p. 132.

${ }^{70}$ Ainsi, en avr. 1342, la reine annonçait au pape qu'elle venait de créer un collège avec la coopération de son chapelain (Highfield, « The Early Colleges », loc. cit., p. 238).

${ }^{71}$ Aston et Faith, « The Endowments... », loc. cit., p. 304-306.
} 
fondateur, la collation des bourses ainsi créées $^{72}$. Par la suite, Charles V acheva l'œuvre de son père en confiant le gouvernement du collège Mignon à son aumônier ${ }^{73}$. Cette procédure de captation, appliquée à une petite fondation, ne manqua pas d'inspirer la politique royale vis-à-vis d'autres établissements séculiers. Ainsi, une intervention comparable eut lieu, vingtcinq ans plus tard, au collège de Maître Gervais, même si, sur le fond, l'opération ressemblait davantage à une cofondation. Médecin successif de Jean le Bon et de Charles V, Gervais Chrétien $(\uparrow 1382)$ fonda, en février 1371, un collège destiné aux étudiants bayeusains ${ }^{74}$. L'institution attira immédiatement les faveurs du roi, qui alla jusqu'à y créer deux bourses de mathématiciens $^{75}$. En avril 1378, à la demande de Gervais Chrétien, le souverain accepta même de devenir le nouveau fondateur du collège et en délégua, là aussi, le gouvernement à son aumônier ${ }^{76}$. Charles $\mathrm{V}$ réalisait donc en une seule fois ce qui avait demandé deux règnes pour le collège Mignon. Ce tournant radical dans la politique des rois de France correspondait précisément au moment où les Valois étendaient leur contrôle sur le collège de Navarre, afin de transformer cette fondation royale en un instrument de propagande dynastique. Dans ce nouveau contexte, les collèges universitaires ne pouvaient plus être considérés comme de simples fondations charitables, laissées à l'initiative des reines. Les troubles provoqués par la guerre de Cent Ans donnèrent même au souverain l'occasion de s'ériger en véritable réformateur des fondations séculières avec l'union des collèges de Reims et de Rethel.

Fondé en 1409, grâce à un legs de l'archevêque Guy de Roye, le collège de Reims s'était installé en 1415 dans l'ancien hôtel de Bourgogne, près de l'église Saint-Hilaire ${ }^{77}$. Ruiné par la guerre civile entre Armagnacs et Bourguignons, l'établissement avait été abandonné, comme beaucoup d'autres, pendant l'occupation anglo-bourguignonne de Paris ${ }^{78}$. C'est en janvier 1444 que Charles VII décida de le refonder et de le réunir au collège de Rethel, qui avait, lui aussi, pratiquement disparu depuis sa fondation par Gautier de Launoy au XIII $^{\mathrm{e}}$ siècle $^{79}$. Même si le choix de ces deux institutions champenoises était explicitement lié à l'intervention du confesseur du roi, Gérard Machet, qui avait déjà participé à la création du collège de Reims, l'ordonnance de 1444 n'en constituait pas moins un dégré supérieur dans l'interprétation par le souverain de la volonté des fondateurs. Par cet acte, Charles VII obligeait en effet deux établissements, qui n'avaient, à l'origine, aucun lien entre eux, à gérer leur patrimoine en commun. Une telle initiative semblait toutefois se justifier par une

\footnotetext{
${ }^{72}$ N. Gorochov, « Crises et conflits de pouvoir dans les collèges parisiens au XIV siècle : l'exemple du collège Mignon (1353-1420) », Bibliothèque de l'École des Chartes, 151 (1993), p. 263-264.

${ }^{73}$ L'aumônier du roi apporte son appui au collège dans un procès au Parlement (1367) et nomme, au nom du roi, l'un des boursiers de la fondation (1371), mais on ne peut pas dater précisément l'origine de cette mise sous tutelle (Gorochov, « Crises et conflits... », loc. cit., p. 266-267).

${ }^{74} \mathrm{P}$. de Longuemare, « Le collège de Maître Gervais Chrétien à Paris », Bulletin de la société des antiquaires de Normandie, 31 (1916), p. 202.

${ }^{75}$ Le principe de cette fondation était déjà inscrit dans la donation par Charles V des dîmes de Senneville en Normandie (10 oct. 1374). Il faut toutefois attendre le 30 oct. 1377 pour que le roi verse les 920 fr. d'or censés entretenir les deux boursiers, « lesquels liront les sciences mathematiques permises a lire en nostre université de Paris » (Longuemare, « Le collège de Maître Gervais... », loc. cit., p. 203-205).

${ }^{76}$ Longuemare, « Le collège de Maître Gervais... », loc. cit., p. 205.

${ }^{77}$ H. Lacaille, «Étude sur le collège de Reims à Paris, 1412-1763 », Travaux de l'Académie nationale de Reims, 104 (1897-1898), t. 2, p. 4-9. Les instruments de fondation du collège ont été édités par L. Le Grand, «Le codicille de Gui de Roye, archevêque de Reims, ses divers legs et la fondation du collège de Reims à Paris ", Travaux de l'Académie nationale de Reims, 97 (1894-1895), t. 1, p. 104-113.

${ }^{78}$ À partir de 1423, le collège ne paie plus à l'abbaye Sainte-Geneviève le cens qu'il doit pour son immeuble (Lacaille, "Étude sur le collège de Reims... », loc. cit., p. 9, n. 2).

${ }^{79}$ Lacaille, «Étude sur le collège de Reims... », loc. cit., p. 10-11. L'ordonnance éditée par Lacaille (ibid., p. 105-107, $\mathrm{n}^{0} 3$ ), d'après une copie fautive du XVI $\mathrm{e}^{\mathrm{e}}$ s., doit être écartée au profit de la version enregistrée au parlement de Paris, le 4 mars 1445, cf. H. Stein, Inventaire analytique des ordonnances enregistrées au parlement de Paris jusqu'à la mort de Louis XII, Paris, 1908, p. 44, nº 638.
} 
nécessité matérielle : la ruine des collèges séculiers perturbait gravement le fonctionnement de l'université tout entière. C'est sans doute la raison pour laquelle le même roi lança, en 1459, une réforme du collège de Navarre, qui avait, lui aussi, été mis à sac lors de la guerre civile. À la restauration du studium, engagée par la réforme d'Estouteville (1452), devait donc correspondre celle des collèges, qui en constituaient la base. Mais, au-delà de ces circonstances proprement parisiennes, il paraît difficile de ne pas voir dans l'activisme de Charles VII une manière de répondre à l'ambitieux programme de son neveu et adversaire dynastique, le roi d'Angleterre, qui venait justement de fonder King's College (1441). Or, pour être l'illustration la plus éclatante de la politique d'Henri VI, cette fondation ne fut pas la seule de ses interventions dans les collèges anglais, puisqu'il participa à la naissance de trois autres établissements entre 1438 et 1448.

La première de ces fondations eut lieu à Oxford et échappa clairement à l'initiative du souverain, tout en constituant un exemple archétypal de cofondation. En mai 1438, au pire moment du conflit franco-anglais, l'archevêque de Cantorbéry, Henry Chichele ( $\uparrow 1443)$, créait All Souls College, au nom évocateur. Les boursiers de cette fondation avaient en effet la mission de prier pour le salut des âmes d'Henri V († 1422), de son frère le duc de Clarence $(\dagger 1421)$ et des Anglais morts à la guerre en France ${ }^{80}$. Principal membre du Conseil, Chichele avait été l'un des artisans de la politique lancastrienne d'exaltation du sentiment national. La dotation de son collège reposait d'ailleurs, en grande partie, sur le rachat des biens des prieurés étrangers confisqués par le roi $^{81}$. On comprend mieux pourquoi l'archevêque de Cantorbéry céda au souverain toute la propriété de l'établissement et fit même établir la charte de fondation au nom du jeune Henri VI, alors âgé de seize ans. Pour Ernest Jacob, ce type de cofondation permettait en fait au fondateur, qui avait doté son collège grâce à des confiscations royales, de se prémunir de tout risque de reprise par la Couronne ${ }^{82}$. Bien que beaucoup plus modeste, la cofondation de Godshouse, à Cambridge, résultait de préoccupations similaires. William Byngham (†1451), curé de St John Zachary à Londres, travaillait, depuis 1436, à un projet de collège ${ }^{83}$. Le site qu'il avait acquis ayant été englobé dans la dotation de King's College, il formula un second projet, qui reçut, en 1446, une autorisation royale ${ }^{84}$. Mais, le 16 avril 1448, Henri VI délivra une nouvelle charte de fondation par laquelle il devenait officiellement le fondateur de Godshouse, à la demande de Byngham, qui recevait en échange le titre de cofondateur ${ }^{85}$. Cette opération évitait en fait que l'institution ne disparaisse prématurément dans la dotation du grand collège royal ${ }^{86}$.

Au même moment, la politique collégiale d'Henri VI s'exprimait aussi par l'intermédiaire de sa jeune épouse française, Marguerite d'Anjou (†1482), à qui 1'on doit la naissance du futur Queens' College à Cambridge. L'initiative en revint à Andrew Dokett († 1484), principal du St Bernard's Hostel, qui obtint en 1446 une licence royale pour fonder un collège Saint-Bernard, non loin de son premier établissement ${ }^{87}$. La dotation se révélant insuffisante,

\footnotetext{
${ }^{80}$ Rashdall, The Universities of Europe..., op. cit., III, p. 226.

${ }^{81}$ Ibid., p. 227.

${ }^{82}$ Jacob, « Founders and Foundations... », loc. cit., p. 158.

${ }^{83}$ Ayant commencé à réunir la dotation dès 1436, William Byngham avait ensuite obtenu plusieurs licences royales, en 1439 et en 1442, pour fonder son collège (A. H. Lloyd, The Early History of Christ's College Cambridge, derived from contemporary documents, Cambridge, 1934, p. 35-40, 50-56).

${ }^{84}$ Lloyd, The Early History..., op. cit., p. 74-76.

${ }^{85}$ Document édité par Lloyd, The Early History..., op. cit., p. 361-372.

${ }^{86}$ Cet établissement ne prit toutefois son véritable essor qu'en mai 1505, lorsque Henri VII autorisa sa mère, Lady Margaret Beaufort $(\uparrow 1509)$, à augmenter considérablement cette première dotation pour fonder Christ's College, cf. B. Dobson, «The Foundation», dans Christ's. A Cambridge College over five Centuries, D. Reynolds éd., Basingstoke, 2005, p. 3-34.

${ }^{87}$ A History of the University of Cambridge, op. cit., I, p. 229.
} 
cette petite structure de quatre boursiers servit alors de base à la fondation scolaire de Marguerite d'Anjou, qui y installa un Queen's College en avril 1448, lui-même refondé en mars 1475 par Élisabeth Woodville $(\dagger$ 1492), qui était devenue reine d'Angleterre après son mariage avec Édouard IV $(1464)^{88}$. L'institution, qui prit alors le nom de Queens' College, pour rendre hommage à ses deux cofondatrices, ne dut toutefois sa survie qu'à la persévérance d'Andrew Dokett, son premier maître, qui participa activement à l'installation du collège de Marguerite d'Anjou et inspira à Élisabeth Woodville les statuts de $1475^{89}$. Mais le plus étonnant est que l'établissement continua à bénéficier des faveurs royales durant le règne de Richard III (1483-1485) ${ }^{90}$. Ainsi, dans une donation de juillet 1484 , le roi précise étrangement qu'il favorise ce collège, parce qu'il aurait été fondé par sa femme, Anne Neville, de fundatione et patronatu prefate consortis nostre, ce qui revenait à faire de Queens' College une fondation permanente des reines d'Angleterre ${ }^{91}$. Une telle politique ne fut cependant poursuivie ni par Henri VII (1485-1509), ni surtout par son épouse, Élisabeth d'York, que le collège laissa totalement indifférente ${ }^{92}$.

La mise en parallèle des interventions royales dans les collèges séculiers de Paris, Oxford et Cambridge dévoile le rapport d'imitation, sinon de concurrence, qui pouvait exister entre les deux couronnes rivales. L'initiative revient sans conteste aux souverains anglais, qui réalisent leurs premières cofondations de collèges sous les règnes d'Édouard II et d'Édouard III. Mais cette politique est suivie de peu par celle de Jean le Bon et Charles V, qui déploient, dès le milieu du XIV ${ }^{\mathrm{e}}$ siècle, un interventionnisme spécifique à l'égard des collèges parisiens. La fin de la guerre de Cent Ans voit, quant à elle, renaître une émulation comparable entre les politiques collégiales d'Henri VI et de Charles VII, ce dernier paraissant, là aussi, répondre à une initiative anglaise. Ce type d'interventions radicales dans des fondations séculières semble d'ailleurs avoir été plus courant en Angleterre : on compte trois refondations ou cofondations royales à Paris contre cinq à Oxford et Cambridge, qui possédaient, on le sait, deux fois moins de collèges. Si la chronologie des interventions rapproche les deux interventionnismes, leur nature, en revanche, les distingue plus nettement. Ainsi, les souverains anglais ont exclusivement pratiqué des cofondations à la demande de particuliers ou avec leur accord. À l'inverse, les rois de France ont, dans deux cas sur trois, procédé à des refondations autoritaires après la mort des fondateurs initiaux. En ce qui concerne la réunion des collèges de Reims et de Rethel, l'ordonnance de 1444 invoquait l'absence d'héritiers, ce qui laissait au roi les mains libres. Mais, dans le cas du collège Mignon, la refondation eut lieu contre l'avis du frère du fondateur, qui était pourtant son principal exécuteur testamentaire. Les exemples anglais suggèrent, de leur côté, une instrumentalisation de l'interventionnisme royal par des fondateurs qui y voient un moyen de pérenniser l'institution. Il faut en effet rappeler qu'Adam of Brome (1326-1332), Robert of Eglesfield (1341-1349), William Byngham (1448-1451) et Andrew Dokett (1448-1484) se sont maintenus, jusqu'à leur mort, à la tête des collèges qu'ils avaient eux-mêmes créés, et ce malgré l'intervention du roi. Sur les sept fondations et cofondations royales anglaises, seul le King's Hall resta sous administration directe de la Couronne, alors que trois des cinq fondations et refondations royales françaises furent placées par Charles V sous l'autorité du

\footnotetext{
${ }^{88}$ Twigg, A History of Queens' College..., op. cit., p. 9.

${ }^{89} \mathrm{Ibid}$., p. 6, 9. Selon D. Leader, Dokett « had a singular and very useful ability to get other people to underwrite the costs of his dreams » (A History of the University of Cambridge, op. cit., I, p. 229).

${ }^{90} \mathrm{La}$ faveur de Richard III ne date pas de son avènement : dès 1477 , le futur roi, alors duc de Gloucester, avait déjà fondé quatre bourses au collège (Twigg, A History of Queens' College..., op. cit., p. 11).

${ }^{91}$ Twigg, A History of Queens' College..., op. cit., p. 11. La dotation de 1484 avait beau inclure un grand nombre de rentes offertes par la reine, cela ne faisait pas, pour autant, de Queens' College une fondation d'Anne Neville.

${ }^{92}$ Twigg, A History of Queens' College..., op. cit., p. 12.
} 
confesseur ou de l'aumônier du roi ${ }^{93}$. En résumé, si les souverains anglais sont intervenus plus souvent et plus tôt dans les collèges séculiers, leurs homologues français ont exercé un contrôle beaucoup plus étroit sur ce type d'établissements. Il va de soi qu'une telle situation n'a pas manqué de peser sur la définition du lien entre le roi et l'université.

\section{Un lien organique entre le pouvoir royal et l'université}

Les trois universités étudiées ne sont pas, à proprement parler, des fondations royales et doivent leur naissance et leur développement tout autant à l'action des papes qu'à celle des rois. Mais, en tant qu'institutions universitaires, les collèges séculiers ont pu, dans certains cas, renforcer le lien entre le souverain et l'université, au point de créer, de toutes pièces, une relation privilégiée entre le prince et son studium.

Ainsi, l'existence d'une telle liaison a pu être évoquée pour justifier une intervention royale au profit d'un collège. Ce fut le cas pour University College, 1'un des plus vieux établissements d'Oxford, qui était empêtré, à la fin du XIV siècle, dans un litige inextricable devant la cour du Banc du roi. L'issue du procès devenant incertaine devant cette juridiction ordinaire, l'université, qui administrait le collège depuis sa fondation, demanda que la cause fût directement jugée par le roi en son conseil privé. Or, dans leur pétition d'avril 1379, les universitaires d'Oxford motivèrent cette démarche par le fait que University College et, par voie de conséquence, l'université tout entière avaient été fondés par Alfred le Grand, roi du Wessex (871-899) et donc prédécesseur du souverain ${ }^{94}$. Cet argument semble avoir reçu un accueil favorable à la chancellerie : dès 1381, un writ fait référence à la fondation royale du collège et, en 1388, le writ de supersedeas, qui réserve la cause au conseil du roi, rappelle, lui aussi, ce mythe de fondation ${ }^{95}$. L'origine mythique de l'université d'Oxford n'avait été évoquée, jusqu'alors, que dans un passage du Polychronicon, rédigé à partir de 1327 par Ranulf Higden ( $\uparrow 1364$ ), moine bénédictin de l'abbaye de Chester ${ }^{96}$. Cette chronique bénéficiait certes d'une très bonne diffusion : on conserve quelque cent dix-huit manuscrits du Polychronicon, ce qui le place en neuvième position dans le classement des œuvres historiques les plus diffusées de tout le Moyen Âge ${ }^{97}$. Elle n'en revêtait pas moins un caractère officieux. Les documents, qui justifient l'intervention royale à University College, semblent donc être les premiers textes qui allèguent officiellement cette origine mythique et admettent surtout son authenticité historique. L'initiative revient ici à l'université, qui, pour attirer la bienveillance royale, invoqua son lien intime, presque organique, avec le souverain, qui devenait ainsi l'unique acteur de la fondation du studium. Les rois d'Angleterre ne semblent pourtant pas avoir fait de ce mythe le lieu commun de leurs relations avec cette université. L'argument est réutilisé par le collège en 1427 dans un autre procès, qui l'opposait

\footnotetext{
93 Il s'agit des collèges Mignon (av. 1371), de Navarre (av. 1372) et de Maître Gervais (1378).

${ }^{94}$ Comme l'indique clairement la requête du collège : «A lour tres excellent et tres redouté et tres sovereyn seigneur, notre seigneur le roy, et a son tres sage conseil monstrent ses povres oratours les mestres et escolers de son college appelez Mokel Université Halle en Oxenford, quele college estoit primierement funduz par votre noble progenitour le roy Alfrid, qi Dieux assoill, pur la sustenance de vyngt et sys dyvins perpetuels [...] Que plese a votre tres sovereyn et gratieus seigneur le roy, depuis que vous estez notre vraie foundoure et avowe, de faire comparoir devant votre tres sage conseil les parties avant dites pur monstrer leur evidences sur le droit de la matire sus dite, issint que a cause del povertee de vos ditz oratours votre dit college ne soit disheritez en maniere surdit» (J. Parker, The Early History of Oxford, 727-1100, preceded by a sketch of the Mythical Origin of the City and University, Oxford, 1885, p. 316, $\mathrm{n}^{\circ} 22$ ).

${ }^{95}$ Parker, The Early History of Oxford..., op. cit., p. 55-56.

${ }^{96} \mathrm{~S}$. Lusignan, «Les mythes de fondations des universités au Moyen Âge », Mélanges de l'École française de Rome. Moyen Âge, 115 (2003), p. 457.

${ }^{97}$ B. Guenée, Histoire et culture historique dans l'Occident médiéval, Paris, 1980, p. 250.
} 
à l'abbaye d'Oseney ${ }^{98}$. Mais il faut attendre le début du XVIII ${ }^{\mathrm{e}}$ siècle pour que la Couronne se le réapproprie et s'en serve pour imposer la tutelle royale sur University College, suivant un jugement de la cour du Banc du roi, qui inscrit donc dans la common law la fondation mythique de l'université d'Oxford ${ }^{99}$.

Du strict point de vue de la politique universitaire, la refondation du collège Mignon par Jean le Bon parait bien plus révolutionnaire. Pour justifier cette intervention royale dans une fondation privée, l'ordonnance de juillet 1353 dut expliciter la relation qui liait le collège au roi. La chancellerie procéda alors à une double redéfinition des liens qui unissaient, d'une part, le collège et l'université et, d'autre part, cette dernière et le souverain. Si les premiers étaient d'ordre juridique, à travers la défense des droits des écoliers, les seconds renvoyaient à une sorte de parenté symbolique, l'université ayant été adoptée par le roi. C'est, en effet, à l'occasion de la refondation du collège Mignon que la chancellerie qualifia, pour la première fois, l'université de Paris de « fille du roi », dilecta filia et fidelis nostra universitas ${ }^{100}$. On pensait jusqu'alors que la première mention de ce lieu commun des relations entre le roi de France et le studium parisien remontait à un jugement de Jean le Bon, rendu au Parlement le 6 septembre $1354^{101}$. En fait, il se trouvait lié, dès l'origine, à l'interventionnisme royal vis-àvis des collèges. Il est important de noter que cette redéfinition modifiait sensiblement la nature même des deux institutions qu'elle concernait. Elle transformait l'université de Paris, non en une fondation, mais en une créature du souverain, qui devenait, de ce fait, son véritable père spirituel. Elle tendait aussi à faire du collège séculier, fondation de nature privée, une institution d'intérêt général, que l'université et bientôt l'État se devaient de favoriser, sinon de contrôler. La réunion autoritaire des collèges de Reims et de Rethel (1444), au nom de l'ordre public, en fut une bonne illustration. On sait par ailleurs qu'au même moment, le parlement de Paris veillait régulièrement au respect des statuts de collèges et n'hésitait pas, le cas échéant, à contrôler leur administration ${ }^{102}$. La justice royale s'arrogeait ainsi le droit de surveiller toute institution censée exercer une «fonction publique », même lorsqu'il s'agissait de fondations privées ${ }^{103}$. La création d'un lien organique entre le roi et l'université de Paris, à travers les collèges, semble donc être la première étape d'une mise au pas de cette corporation, qui finit, $\mathrm{au} \mathrm{XV}^{\mathrm{e}}$ siècle, par se soumettre au droit commun du royaume ${ }^{104}$.

L'utilisation du collège comme passerelle juridique entre le prince et son studium présente des formes distinctes en France et en Angleterre. Dans le cas d'Oxford, c'est l'université qui prend l'initiative de créer ce lien en officialisant le mythe d'une fondation royale. Dans le cas de Paris, c'est au contraire le souverain qui décide d'adopter l'université comme sa fille. Or, il y a une différence fondamentale entre un roi-père et un roi-fondateur, car le lien de fondation

\footnotetext{
${ }^{98}$ Lusignan, « Les mythes de fondations... », loc. cit., p. 463.

${ }^{99}$ Rashdall, The Universities of Europe..., op. cit., III, p. 179.

${ }^{100}$ Selon les propres termes de la charte de refondation : «Johannes, Dei gracia Francorum rex, notum facimus universis tam presentibus quam futuris quod [...] dilecta filia et fidelis nostra universitas scolarium parisiensium studentium ad quam, ut asserit, jura, libertates et franchisias scolarium ejusdem quorumcumque et eorum fundationes et redditus quoscumque prosequi dinoscitur pertinere, dilectum et fidelem magistrum Robertum Mignon, clericum nostrum dicte Camere compotorum fratremque ac supremum exequtorem defuncti memorati [Jean Mignon], ob fundationem predictam, coram nobis conveniri et suam petitionem super hoc fieri fecisset 》 (P. Guyard, « La fondation du collège Mignon », Bibliothèque de l'École des Chartes, 151 (1993), p. 286).

${ }^{101}$ Il s'agissait d'une affaire qui opposait l'université au prévôt de Paris, Guillaume Staise, et à l'avocat du roi, Renaud d'Acy (S. Lusignan, "Vérité garde le roy». La construction d'une identité universitaire en France (XIII $I^{e}-X V^{e}$ siècle), Paris, 1999, p. 267).

${ }^{102} \mathrm{~K}$. Weidenfeld, Les origines médiévales du contentieux administratif (XIV -XV siècles), Paris, 2001, p. $152-153$.

${ }^{103}$ Ibid., p. 154.

${ }^{104}$ Lusignan, « Vérité garde le roy »..., op. cit., p. 280-281.
} 
est beaucoup moins contraignant que la filiation adoptive. Les droits du fondateur sont en principe fixés par l'acte même de fondation, ce qui les réduit à néant dans le cas d'une origine mythique. La plupart de ces mythes bénéficiaient ainsi aux institutions fondées, qui pouvaient toujours se prévaloir de l'ancienneté et de l'honorabilité de leurs prétendus fondateurs, sans en subir la tutelle. Les souverains anglais ne s'y sont d'ailleurs pas trompés, puisqu'ils ont mis plus de trois siècles à revendiquer une telle relation. Il en va tout autrement de la paternité instituée par l'adoption: dès la fin du XII siècle, les juristes admettaient que cette parenté adoptive devait être assimilée à la parenté naturelle par une fiction du droit ${ }^{105}$. Or, nul n'est besoin de texte pour définir l'étendue de la puissance paternelle. On peut donc dire qu'à Oxford, le lien organique créé par le collège revenait à instrumentaliser la relation du studium avec le souverain, alors qu'à Paris, il constituait, dès le départ, un instrument de coercition entre les mains du roi. En somme, la liaison établie entre ces universités et la Couronne se révélait déterminée par la nature même des liens - autoritaires en France et négociés en Angleterre - que celle-ci entretenait avec les collèges universitaires. Il semble donc que se soit constitué, dans la seconde moitié du XIV siècle, une triade fonctionnelle réunissant le roi, le collège et l'université, au sein de laquelle la relation entre deux pôles influençait nécessairement leur rapport au troisième. Ainsi, en dépit d'une pratique différente de l'interventionnisme royal dans les collèges séculiers de Paris, Oxford et Cambridge, ces derniers jouèrent un rôle identique, de part et d'autre de la Manche, dans le système institutionnel reliant 1'État aux universités à la fin du Moyen Âge.

Thierry Kouamé

Université Paris 1 Panthéon-Sorbonne

Laboratoire de médiévistique occidentale de Paris (UMR 8589)

${ }^{105}$ Fr. Roumy, L'adoption dans le droit savant du XII au XVI' siècle, Paris, 1998, p. 116-119. 